\title{
Effects of hyperbaric oxygen on the Nrf2 signaling pathway in secondary injury following traumatic brain injury
}

\author{
X.E. Meng, Y. Zhang, N. Li, D.F. Fan, C. Yang, H. Li, D.Z. Guo and S.Y. Pan \\ Department of Hyperbaric Oxygen, Navy General Hospital, Beijing, China \\ Corresponding author: S.Y. Pan \\ E-mail: shuyipan_oooo@126.com \\ Genet. Mol. Res. 15 (1): gmr.15016933 \\ Received August 10, 2015 \\ Accepted October 30, 2015 \\ Published January 29, 2016 \\ DOI http://dx.doi.org/10.4238/gmr.15016933
}

ABSTRACT. We investigated the effects of hyperbaric oxygen treatment on the Nrf2 signaling pathway in secondary injury following traumatic brain injury, using a rat model. An improved Feeney freefall method was used to establish the rat traumatic brain injury model. Sixty rats were randomly divided into three groups: a sham surgery group, a traumatic brain injury group, and a group receiving hyperbaric oxygen treatment after traumatic brain injury. Neurological function scores were assessed at 12 and $24 \mathrm{~h}$ after injury. The expression levels of Nrf2, heme oxygenase 1 (HO-1), and quinine oxidoreductase 1 (NQO-1) in the cortex surrounding the brain lesion were detected by western blotting $24 \mathrm{~h}$ after the injury. Additionally, the TUNEL method was used to detect apoptosis of nerve cells $24 \mathrm{~h}$ after traumatic injury and Nissl staining was used to detect the number of whole neurons. Hyperbaric oxygen treatment significantly increased the expression of nuclear Nrf2 protein ( $P<0.05), \mathrm{HO}-1$, and NQO-1 in the brain tissues surrounding the lesion after a traumatic brain injury $(P<0.05)$ and also significantly reduced the number of apoptotic and injured nerve cells. The neurological function scores also improved with hyperbaric oxygen treatment $(P<0.05)$. Therefore, hyperbaric oxygen has a neuroprotective 
role in traumatic brain injury, which is mediated by up-regulation of the Nrf2 signaling pathway.

Key words: Traumatic brain injury; Hyperbaric oxygen; Nrf2

\section{INTRODUCTION}

Traumatic brain injury produces high morbidity, mortality, and disability rates worldwide (Lannin et al., 2014), and it is a major threat to human life and health. However, effective therapeutic treatments for traumatic brain injury in clinical practice are limited. Many previous studies have shown that hyperbaric oxygen therapy effectively improves the prognosis in patients with traumatic brain injury; however, the specific protective mechanisms of hyperbaric oxygen have not been completely elucidated. The present study aimed to increase understanding of the mechanisms of hyperbaric oxygen treatment following traumatic brain injury by examining the effects of hyperbaric oxygen on the Nrf2 signaling pathway. Increased knowledge of signaling mechanisms will contribute to improved efficacy of hyperbaric oxygen therapy in clinical practice.

\section{MATERIAL AND METHODS}

\section{Animals and group assignment}

Sixty healthy adult male Sprague Dawley rats were randomly divided into three groups: a sham surgery group, a traumatic brain injury group, and group with traumatic brain injury receiving hyperbaric oxygen treatment. An improved Feeney method was used to establish the rat traumatic brain injury model. Chloral hydrate $(4 \mathrm{mg} / \mathrm{kg})$ was used to anaesthetize the rats before they were fixed on a stand. After skin preparation and disinfection, an orthopedic drill was used to make a $5-\mathrm{mm}$ diameter bone fenestration at $3 \mathrm{~mm}$ to the right of the coronary suture midline and $3 \mathrm{~mm}$ behind the sagittal suture, while keeping the dura intact. A 40-g object was allowed to free-fall from a height of $15 \mathrm{~cm}$ and vertically strike the right dura mater, causing an injury with a depth of $3 \mathrm{~mm}$ and a diameter of $4 \mathrm{~mm}$. The sham surgery group underwent only the fenestration treatment.

\section{Hyperbaric oxygen therapy}

Hyperbaric oxygen therapy was administered as described in previous reports (Wang et al., 2010). Hyperbaric oxygen treatment was initiated within $2 \mathrm{~h}$ of traumatic brain injury. Rats in the hyperbaric oxygen treatment group were placed in a chamber and the chamber was flushed with pure oxygen for $10 \mathrm{~min}$, in order to achieve a final oxygen concentration of $>95 \%$. Then, the pressure was increased at a constant rate to $0.12 \mathrm{MPa}$ (gauge pressure), which was maintained for $60 \mathrm{~min}$. Next, the pressure was gradually decreased to atmospheric pressure at a constant rate, over a 20 -min period. In total, two treatments were conducted within a 10-h period. The behavior of the rats was closely monitored while they were in the chamber. Animals in the sham surgery and traumatic brain injury groups were placed in the pressurized chambers, in order to simulate experimental processes and environmental conditions other than pressure and oxygen concentration. 


\section{Western blot assay}

Rats were anaesthetized $24 \mathrm{~h}$ after injury and perfused from the apex of the heart using 100 $\mathrm{mL}$ saline. The brain tissue surrounding the lesion was removed and stored in a $-80^{\circ} \mathrm{C}$ freezer until use. Extraction of nuclear and cytoplasmic proteins was performed according to the kit instructions and protein concentrations were determined using the Bradford method. Samples were mixed with $5 \mathrm{X}$ loading buffer at a 1:4 ratio and boiled for $10 \mathrm{~min}$ to denature the proteins. Protein (35 $\mu \mathrm{g}$ ) was loaded into each well for electrophoresis. After membrane transfer, the samples were blocked for one h. Diluted primary antibodies against Nrf2, heme oxygenase 1 (HO-1), NADPH, quinine oxidoreductase 1 (NQO1), $\beta$-actin, and H3 (1:1000 dilution, all purchased from Abcam Co., Cambridge, MA, USA) were added, and the membranes were incubated overnight on a shaker at $4^{\circ} \mathrm{C}$. After incubation with the secondary antibody, membranes were washed. An enhanced chemiluminescent reagent was added to develop the film, followed by gray-scale analyses using the Image J software.

\section{Nissl staining analysis}

The Nissl staining procedure was performed according to procedures described previously (Zhuang et al., 2012).

\section{Detection of apoptosis by the TUNEL method}

Paraffin-embedded brain tissue was sectioned into 4-mm thick slices, using a microtome. A TUNEL assay was used to detect apoptosis, according to the manufacturer instructions (Roche, USA). TUNEL results for the areas surrounding the lesions were observed under a light microscope. Ten fields at 400X magnification were randomly selected and the apoptotic index was calculated based on the percentage of TUNEL positive cells compared to the total number of cells.

\section{Post-traumatic neurological function scoring}

Post-traumatic neurological function scores of rats were determined using the neurological severity scores method (Si et al., 2014), which mainly includes motor function, sensory function, and balance ability tests, as well as assessments of physiological reflex defects and abnormal movements; the maximum possible score is 18 points. A score of one point was assigned when rats could not perform the task or did not provide an appropriate response. A score of 13-18 points was considered an indication of severe injury, 7-12 points was considered moderate injury, and 1-6 points was considered mild injury.

\section{Statistical analyses}

The SPSS 15.0 software was used for data processing (SPSS, IBM, Armonk, NY, USA). Data are reported as means \pm standard deviations (means $\pm \mathrm{SD}$ ). A Kruskal-Wallis test was used to analyze neurological function score data. One-way analysis of variance was used for other comparisons between multiple groups. 


\section{RESULTS}

\section{Effects of hyperbaric oxygen therapy on neurological function scores of rats with traumatic brain injury}

Compared to the sham surgery group, the neurological function score of the traumatic brain injury group was significantly decreased (Table $1 ; P<0.05)$. Hyperbaric oxygen treatment significantly improved the loss of neurological function loss in rats with traumatic brain injury (Table $1 ; P<0.05$ ).

Table 1. Neurological function scores at 12 and $24 \mathrm{~h}$.
\begin{tabular}{l|c|c|c}
\hline Group & $\mathrm{N}$ & $12 \mathrm{~h}$ (means \pm SD) & $24 \mathrm{~h}(\mathrm{means} \pm \mathrm{SD})$ \\
\hline Sham surgery & 20 & $1.2 \pm 0.03$ & $1.4 \pm 0.01$ \\
\hline Traumatic brain injury & 20 & $14.65 \pm 3.16$ & $15.61 \pm 2.36^{*}$ \\
\hline Hyperbaric oxygen therapy + injury & 20 & $13.02 \pm 1.95$ & $13.21 \pm 1.65^{\#}$ \\
\hline
\end{tabular}

${ }^{*} \mathrm{P}<0.05$ compared to the sham surgery group. $\mathrm{*} P 0.05$ compared to the traumatic brain injury group.

\section{Effects of hyperbaric oxygen on the expression of Nrf2, HO-1, and NQO1}

Compared to the sham surgery group (Figures 1 and 2 and Table 2), the expression levels of Nrf2, $\mathrm{HO}-1$, and NQO1 proteins in brain tissue surrounding the lesion of the traumatic injury group were significantly increased $(P<0.05)$. Hyperbaric oxygen treatment further increased the expression levels of Nrf2, HO-1, and NQO1 proteins $(P<0.05)$, compared to traumatic injury without treatment.

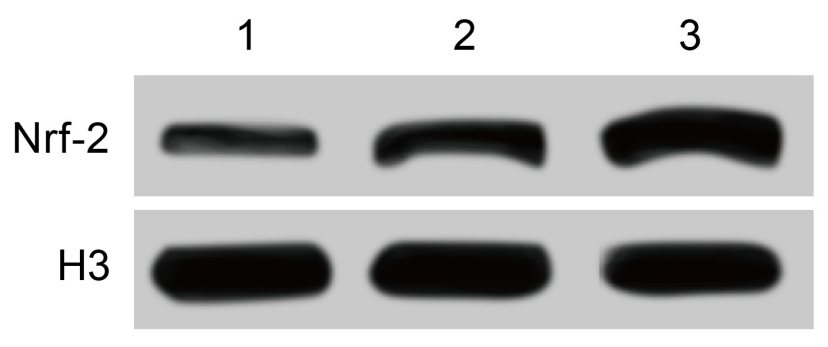

Figure 1. Expression of Nrf2 protein in each group. Lane $1=$ Sham surgery group; lane 2 = traumatic brain injury group; lane 3 = hyperbaric oxygen treatment group.

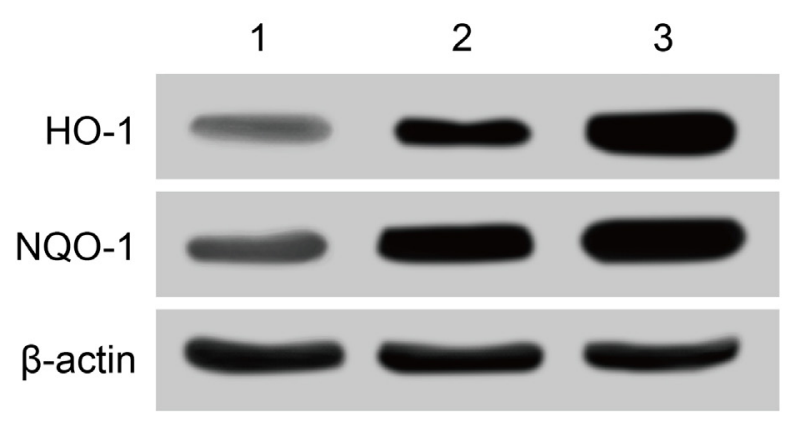

Figure 2. Expression of HO-1 and NQO1 proteins in each group. Lane 1 = Sham surgery group; lane 2 = traumatic brain injury group; lane $3=$ hyperbaric oxygen treatment group. 
Table 2. Analyses of Nrf2, HO-1, and NQO1 expression, as well as the apoptotic indexes.

\begin{tabular}{|c|c|c|c|c|c|c|}
\hline Group & $\mathrm{N}$ & $\mathrm{Nr}+2$ & $\mathrm{HO}-1$ & & Apoptotic index (\%) & Number of whole neurons \\
\hline Sham surgery & 10 & $0.89 \pm 0.37$ & $0.09 \pm 0.01$ & $0.02 \pm 0.01$ & $1.1 \pm 0.2$ & $58.1 \pm 4.6$ \\
\hline Traumatic brain injury & 10 & $0.19 \pm 0.11^{*}$ & $0.63 \pm 0.25^{*}$ & $0.84 \pm 0.45^{*}$ & $30.1 \pm 6.4$ & $6.7 \pm 5.3^{*}$ \\
\hline Hyperbaric oxygen therapy + injury & 10 & $0.69 \pm 0.31 \#$ & $0.12 \pm 0.08^{\#}$ & $0.22 \pm 0.12^{\#}$ & $15.2 \pm 4.1$ & $35.1 \pm 7.8^{\#}$ \\
\hline
\end{tabular}

${ }^{*} \mathrm{P}<0.05$ compared to the sham surgery group, ${ }^{\#} \mathrm{P}<0.05$ compared to the traumatic brain injury group.

\section{Effects of hyperbaric oxygen treatment on apoptosis of neurons surrounding the lesion in rats with traumatic brain injury}

The nuclei of normal neurons were large, round, and colorless. In the sham surgery group, apoptotic neurons were either not observed or rarely observed, while the number of apoptotic neurons was significantly increased in the traumatic brain injury group (Figure 3 and Table 2; $P<0.05)$. However, the number of apoptotic neurons surrounding the lesion was significantly decreased in rats treated with hyperbaric oxygen $(P<0.05)$. Apoptotic neurons were positive in TUNEL staining, showing brown pyknotic nuclei (Figure 3; $\mathrm{P}<0.05$ ).
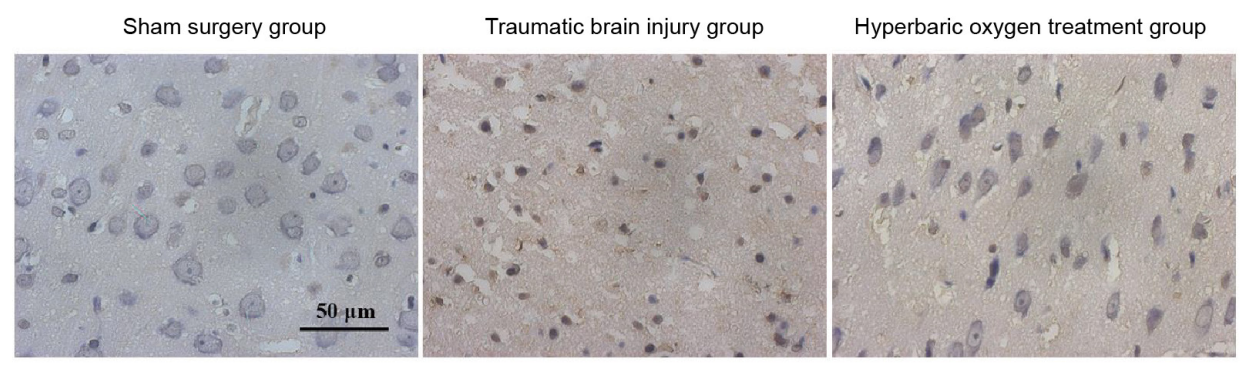

Figure 3. TUNEL staining results for each group.
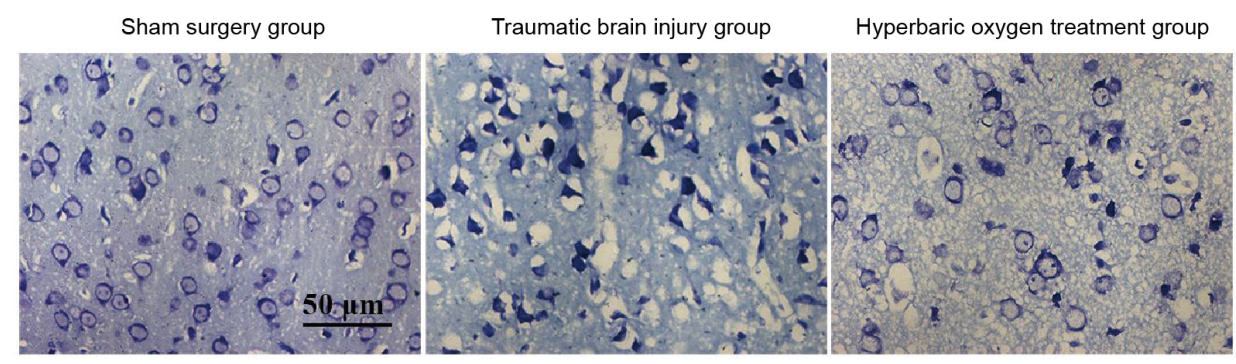

Figure 4. Nissl staining of rat brain tissue from each group.

\section{NissI staining results}

In the sham surgery group, the neurons were large and round. In the traumatic injury group, many neurons were condensed, and the number of whole neurons was significantly reduced (Figure 4; $\mathrm{P}<0.05$ ). The number of condensed neurons surrounding the lesion was significantly reduced in the hyperbaric oxygen treatment condition, and the number of whole neurons was significantly increased compared to the traumatic brain injury group (Figure $4 ; \mathrm{P}<0.05$ ). 


\section{DISCUSSION}

The major mechanisms of traumatic brain injury include primary and secondary brain injury, but the current research focus is on secondary brain injury (Schwab et al., 2014). Nrf2 is a member of the basic leucine zipper protein family. Under physiological conditions, Nrf2 primarily binds to the cytoplasmic adaptor protein Keap1, thereby forming a complex that remains in the cytoplasm. When the cell is stimulated by exogenous substances, Nrf2 is phosphorylated and uncouples from Keap1. Nrf2 protein then translocates from the cytoplasm to the nucleus and binds to the antioxidant responsive element sequence in the promoter region of the target genes, thereby inducing transcription and translation. These gene products include anti-inflammatory proteins, antioxidant enzymes, growth factors, and proteins related to regulation of calcium homeostasis, which further induce the target genes to express $\mathrm{HO}-1, \mathrm{NQO} 1$, and other antioxidant and detoxification enzymes (Hong et al., 2010). These antioxidant and detoxifying factors encompass many important protective mechanisms for secondary brain injury following traumatic brain injury (Miller et al., 2014; Sandberg et al., 2014). Previous studies have found that expression of Nrf2 begins to increase $2 \mathrm{~h}$ after traumatic brain injury, and its expression peaks after $24 \mathrm{~h}$. Nrf2 expression then gradually decreases and returns to normal levels 3 to 5 days later (Cheng et al., 2013). The degree of traumatic brain injury in Nrf2 knockout mice is significantly more severe compared to wild type mice, and administration of drugs that up-regulate Nrf2 protein expression significantly reduces apoptosis of neurons and improves neurological function after traumatic brain injury (Ding et al., 2014; Xie et al., 2014; Xu et al., 2014). Therefore, these results demonstrate that Nrf2 is a participant in secondary brain injury after traumatic brain injury, and regulation of the Nrf2 signaling pathway increases the levels of downstream antioxidants and detoxification enzymes, thereby contributing to neuroprotection.

The results of the present study demonstrate that the expression of Nrf2 protein in the tissue surrounding traumatically injured brain lesions is significantly increased $24 \mathrm{~h}$ after the injury, consistent with previous studies. Hyperbaric oxygen treatment further up-regulated Nrf2 protein expression in the nucleus and further decreased Keap1 protein expression. Additionally, downstream HO-1 and NQO1 were significantly increased following traumatic brain injury, and hyperbaric oxygen treatment further increases their expression. Furthermore, hyperbaric oxygen significantly reduced neuron apoptosis and improved neurological function, indicating that upregulation of the Nrf2 signaling pathway may be one of the mechanisms for the neuroprotective effects of hyperbaric oxygen treatment.

In summary, hyperbaric oxygen treatment up-regulates Nrf2 expression following traumatic brain injury and regulates expression of a series of downstream products. Therefore, the results support the Nrf2 signaling pathway as a mechanism for the neuroprotective effects of hyperbaric oxygen treatment.

\section{Conflicts of interest}

The authors declare no conflicts of interest.

\section{ACKNOWLEDGMENTS}

The authors wish to express their gratitude to the reviewers for their critical comments. 


\section{REFERENCES}

Cheng ZG, Zhang GD, Shi PQ and Du BS (2013). Expression and antioxidation of Nrf2/ARE pathway in traumatic brain injury. Asian Pac. J. Trop. Med. 6: 305-310. http://dx.doi.org/10.1016/S1995-7645(13)60061-9

Ding K, Wang H, Xu J, Li T, et al. (2014). Melatonin stimulates antioxidant enzymes and reduces oxidative stress in experimental traumatic brain injury: the Nrf2-ARE signaling pathway as a potential mechanism. Free Radic. Biol. Med. 73: 1-11. http:// dx.doi.org/10.1016/j.freeradbiomed.2014.04.031

Hong Y, Yan W, Chen S, Sun CR, et al. (2010). The role of Nrf2 signaling in the regulation of antioxidants and detoxifying enzymes after traumatic brain injury in rats and mice. Acta Pharmacol. Sin. 31: 1421-1430. http://dx.doi.org/10.1038/ aps.2010.101

Lannin NA, Laver K, Henry K, Turnbull M, et al. (2014). Effects of case management after brain injury: a systematic review. NeuroRehabilitation 35: 635-641.

Miller DM, Wang JA, Buchanan AK and Hall ED (2014). Temporal and spatial dynamics of Nrf2-antioxidant response elements mediated gene targets in cortex and hippocampus after controlled cortical impact traumatic brain injury in mice. $J$. Neurotrauma 31: 1194-1201. http://dx.doi.org/10.1089/neu.2013.3218

Sandberg M, Patil J, D'Angelo B, Weber SG, et al. (2014). NRF2-regulation in brain health and disease: implication of cerebral inflammation. Neuropharmacology 79: 298-306. http://dx.doi.org/10.1016/j.neuropharm.2013.11.004

Schwab JM, Zhang Y, Kopp MA, Brommer B, et al. (2014). The paradox of chronic neuroinflammation, systemic immune suppression, autoimmunity after traumatic chronic spinal cord injury. Exp. Neurol. 258: 121-129. http://dx.doi.org/10.1016/j. expneurol.2014.04.023

Si D, Li J, Liu J, Wang X, et al. (2014). Progesterone protects blood-brain barrier function and improves neurological outcome following traumatic brain injury in rats. Exp. Ther. Med. 8: 1010-1014.

Wang GH, Zhang XG, Jiang ZL, Li X, et al. (2010). Neuroprotective effects of hyperbaric oxygen treatment on traumatic brain injury in the rat. J. Neurotrauma 27: 1733-1743. http://dx.doi.org/10.1089/neu.2009.1175

Xie Y, Zhao QY, Li HY, Zhou X, et al. (2014). Curcumin ameliorates cognitive deficits heavy ion irradiation-induced learning and memory deficits through enhancing of Nrf2 antioxidant signaling pathways. Pharmacol. Biochem. Behav. 126: 181-186. http://dx.doi.org/10.1016/i.pbb.2014.08.005

Xu J, Wang H, Ding K, Zhang L, et al. (2014). Luteolin provides neuroprotection in models of traumatic brain injury via the Nrf2ARE pathway. Free Radic. Biol. Med. 71: 186-195. http://dx.doi.org/10.1016/j.freeradbiomed.2014.03.009

Zhuang Z, Zhou ML, You WC, Zhu L, et al. (2012). Hydrogen-rich saline alleviates early brain injury via reducing oxidative stress and brain edema following experimental subarachnoid hemorrhage in rabbits. BMC Neurosci. 13: 47. http://dx.doi. org/10.1186/1471-2202-13-47 